\title{
Green Synthesis and Characterization of Nano Phosphorus Fertilizer for Wheat
}

\author{
Sagar Shashikant Patil*, S. S. Balpande, Mohammad Sajid and M. R. Pandao \\ Soil Science and Agricultural Chemistry Section, College of Agriculture, Nagpur, \\ Maharashtra, India \\ *Corresponding author
}

\begin{abstract}
A B S T R A C T
Keywords

Nano P fertilizer,

Zeta potential, Kcps and Polydispersity index

\section{Article Info}

Accepted:

04 November 2020

Available Online:

10 December 2020

Nano phosphorus fertilizer is prepared by chemical precipitation method and characterized with the help of Dynamic Light Scattering and Fourier Transform Infrared Spectroscopy (FTIR). The synthesised nano particles with Pluronic F-68 as a stabilizing agent showed a good size and stability in the suspension.A particle size of $145-172 \mathrm{~nm}$, the Poly-dispersity Index in all the samples range from 0.274 to 0.328 , indicating that these are midrange values and the particles remain in disperse form in all the samples. The zeta potential values of synthesized HAP NPs are found to be -47.13 $\mathrm{mV}$ revealing the better stability in aqueous suspension.

\section{Introduction}

Nanofertilizers are the important tools in agriculture to improve crop growth, yield and quality parameters with increase nutrient use efficiency, reduce wastage of fertilizers and cost of cultivation. The main reason for high interest in nanofertilizers is mainly their penetration capacity; size and very high surface area which is usually differ from the same material found in bulk form. Nanofertilizers are not only ecofriendly but also reduce environment pollution. Nanofertilizers are highly effective for precise nutrient management in precision agriculture

with matching the crop growth stage for nutrient and may provide nutrient throughout the crop growth period. Nano-fertilizers increase crop growth up to optimum concentrations further increase in concentration may inhibit the crop growth due to the toxicity of nutrient. Nanofertilizers were very effective in nutrient uptake (Singh et al., 2017). Nanofertilizers have limitations like synthesis and characterization of nanofertilizer is difficult job and it is less available in market. Nanofertilizer has some disadvantages like it is costlier in market and higher dose of any nanofertilizer led to decrease in yield.
\end{abstract}


Response of $\mathrm{P}$ fertilizer in Vertisols of India has been reported to be unpredictable and this has been ascribed to high $\mathrm{P}$ fixation due to high clay content and high content of smectite. At present $5 \%$ of the Indian soils have adequate available P, $49.3 \%$ Under low category, $48.8 \%$ under medium and only $1.9 \%$ under high category only $20-25 \%$ of applied $\mathrm{P}$ is available for crops and remaining part of converted into insoluble into $\mathrm{P}$ solubilisation of inorganic phosphorous in soil is mostly mediated by microbial activity due to secretion of organic acids like oxalic, malic, citric, acetic lactic, gluconic acid, butyric acid, proponic acid, release during decomposition and by PSB which are the major solubilizes of phosphorous from resources (Pattnayak et al., 2009).

\section{Materials and Methods}

Nano $\mathrm{P}$ fertilizers were prepared using Hydroxyapatite (HAP) via. Chemical precipitation method in the laboratory as demonstrated by Mateus et al., 2007.

After the preparation of hydroxyapatite nanoparticles different characterization techniques were used to investigate their particle size (nm), poly dispersive index (PDI) and count rate (Kcps), zeta potential and morphological characteristics. Dynamic light scattering (Malvern Zetasizer, Nano ZS 90) was used to check particle size (nm), polydispersity index (PDI) and count rate (Kcps) following the standard operating procedure at $25^{\circ} \mathrm{C}$. It is a laser diffraction method with a Multiple Scattering Technique which was used to determine the particle size distribution of the nanoparticles. In order to find out the particle size and particle size distribution from the synthesized nanoparticles in aqueous media, $1 \mathrm{~mL}$ of the suspension was taken in the cuvette and measurements were conducted at $25^{\circ} \mathrm{C}$. Fourier-transform infrared spectroscopy (FTIR) (Perkin Elmer, Spectrum
II) was used to check functional group present on the surface of hydroxyapatite nanoparticles by applying infrared radiation (IR) to samples of materials, FTIR analysis measures a samples absorbance of infrared light at various wavelengths to determine the materials molecular composition and structure. Potassium bromide $(\mathrm{KBr})$ pellet preparation was carried out using $1 \mathrm{mg}$ of dried hydroxyapatite nanoparticles synthesized at $\mathrm{pH} 9.5$ and $100 \mathrm{mg}$ of $\mathrm{KBr}$ and pellet was prepared using $\mathrm{KBr}$ press. The pellet was later subjected to FTIR Perkin Elmer Spectrum II spectroscopy for analysis. It scans in the range of $400-4000 \mathrm{~cm}^{-1}$ were collected for each spectrum at a spectral resolution of $4 \mathrm{~cm}^{-1}$.

\section{Results and Discussion}

\section{Characterization of nano $\mathbf{P}$ particles}

Average particle size and poly-dispersity index of the HAP NPs synthesized via wet chemical method, was evaluated of $8000 \mathrm{ppm}$ of suspensions prepared in deionized Milli-Q water for two spraying schedules using Malvern Zeta Sizer, ZS-90 instrument. Dynamic light scattering (DLS) analyze the velocity of particle movement by measuring dynamic fluctuations of light scattering intensity, caused by the random motion of the particle.

This technique yields an average particle size, poly-dispersity index and counts rate of the particle present in the solution(Murdock et al., 2008). Data presented in table 1 and fig. 1 and fig. 2 revealed that the prepared HAP NPs for two spray schedules showed a particle size of 145-172 nm. The PDI (Poly-dispersity Index) scale in all the samples range from 0.274 to 0.328 which indicate that it is the mid-range value and the particles remain in disperse form in all the samples. 
Table.1 Particle size (nm), PDI and Kcps of synthesized nano P particles

\begin{tabular}{|c|l|c|c|c|}
\hline Sr. No. & \multicolumn{1}{|c|}{ Sample details } & Particle size (nm) & PDI & Kcps \\
\hline $\mathbf{1}$ & $\begin{array}{l}\text { HAP NPs for } 1^{\text {st }} \\
\text { spraying }\end{array}$ & 147.3 & 0.274 & 205.8 \\
\hline $\mathbf{2}$ & $\begin{array}{l}\text { HAP NPs for } 2^{\text {nd }} \\
\text { spraying }\end{array}$ & 172 & 0.328 & 232.9 \\
\hline
\end{tabular}

Fig.1 Particle size distribution of synthesized HAP NPs using dynamic light scattering $1^{\text {st }}$ foliar spray

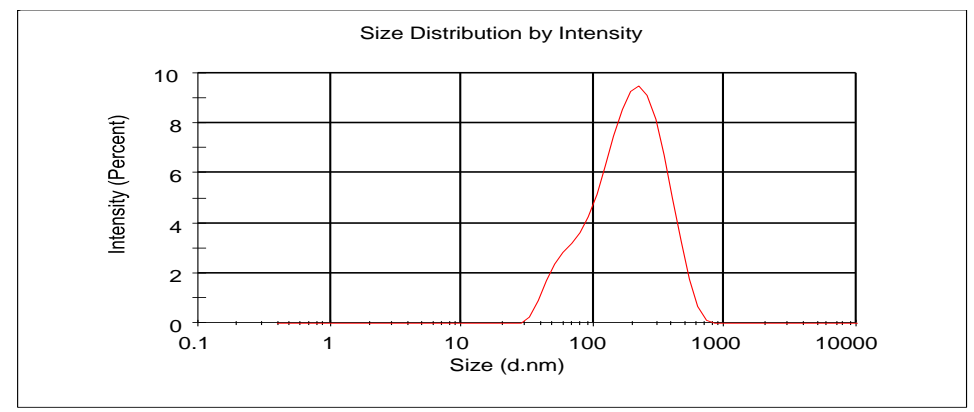

Fig.2 Particle size distribution of synthesized HAP NPs using dynamic light scattering $2^{\text {nd }}$ foliar spray

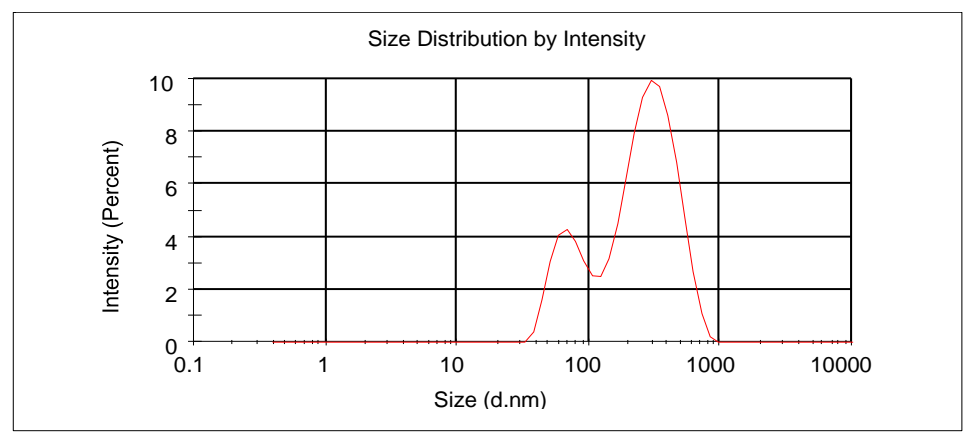

Fig.3 Zeta potential $(\mathrm{mV})$

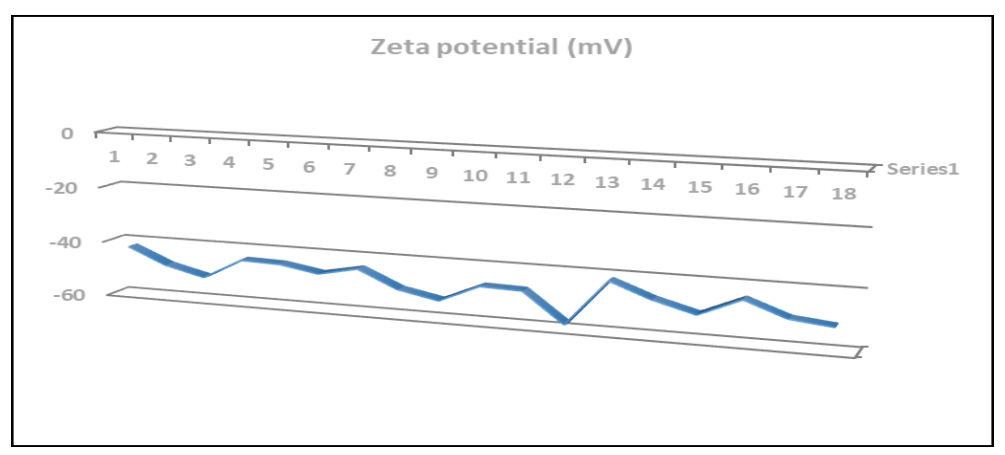


Surface charge of synthesized HAP NPs was measured following standard operating procedure using Malvern Zeta Sizer, ZS-90 instrument. Zeta potential measurement specifies the electro kinetic potential of a colloidal system (Garcia et al., 1997).

Magnitude of the zeta potential is an indicator of repulsive forces between particles and therefore it can provide a good estimation of the suspension stability (Hunter, 1981). The larger zeta potential values represent lower degree of aggregation that leads to higher degree of stability of nanoparticles and smaller Z-averaged hydrodynamic diameter. At lower zeta values, the nanoparticles flocculate early and the stability in nanosuspension reduces.

The common dividing line between unstable and stable suspensions is taken as +30 or -30 $\mathrm{mV}$; particles having zeta potentials beyond these limits are generally considered as stable (Zak et al., 2011). The zeta potential values of synthesized HAP NPs at synthesis are found to be $-47.13 \mathrm{mV}$ as shown in fig. 3, revealing the better stability of synthesized HAP NPs in aqueous suspension.

To determine the functional groups responsible for the synthesis of phosphorous NPs Fourier-transform infrared spectroscopy (FTIR) analysis was performed. FTIR analysis reveals the presence of $-\mathrm{OH}$ and $\mathrm{P}-\mathrm{O}$ groups at 3435.12 and $1034.5 \mathrm{~cm}^{-1}$, respectively. This result is in a good agreement with other works (Hong et al., 2009 and Zak et al., 2011).

In conclusion the study on synthesis of Nano Pfertilizer using Hydroxyapatite (HAP)by chemical precipitation method given by Mateus et al., (2007), revealed that good size and stability of nano $\mathrm{P}$ fertilizer is achieved using Pluronic F-68 as a stabilizing agent.

\section{Acknowledgements}

The author gratefully acknowledge the cooperation and guidance rendered by Director of Research (Agri.), A.A.U., Anand, Assistant Research Scientist and Head, Department of Nanotechnology and Centre for Advanced Research in Plant Tissue Culture, A. A. U., Anand and all the staff members of his office.

\section{References}

Garcia, A. B., Cuesta, A., Montes-Moran, M. A., Martinez-Alonso, A. and Tascon, J. M. D. (1997).Zeta potential as a tool to characterize plasma oxidation of carbon fibres.J. of colloid and interface sci. 19: 363-367.

Hong, R. Y., Li, J. H., Chen, L. L., Liu, D. Q., Li, H. Z., Zheng, Y. and Ding, J. (2009). Synthesis, surface modification and photocatalytic property of $\mathrm{ZnO}$ nanoparticles. Powder Tech. 189: 426432.

Hunter, R. J. (1981). Zeta Potential in Colloid Science: Principles and Applications. Academic Press. 2: 1-386.

Mateus, AYP, M.P. Ferraz and F.J. Monterio, 2007.Comparative study of nanohydroxyapatite microspheres for medical applications. Inc. J. Biomed Mater. Res. 86A: 483-493.

Murdock, R. C., Braydich-Stolle, L., Schrand, A. M., Schlager, J. J. and Hussain, S. B. (2008).Characterization of nanomaterial dispersion in solution prior to In vitro exposure using dynamic light scattering technique. Toxicological Sci. 101(2): 239-253.

Pattnyak, S. K., P. Sureshkumar, J. C. Tarafdar, 2009. New vista in phosphorous research. J. Indian. Soc. Soil Sci. 57(4): 536-545.

Singh M. D., Gautam Chirag, Patidar Om Prakash, Prakasha G. and Vishwajith, 2017.Nanoferilizer is a New Way to 
Increase Nutrient Use Efficiency in Crop Production. Int. J. of Agril. Sci. 9(7): 3831-3833.

Zak, A. K., Razali, R., Majid, W. and
Darroundi, M. (2011). Synthesis and characterization of a narrow size distribution of $\mathrm{ZnO}$ nanoparticles. Int. $J$. of Nanomedicine, 6: 1399-1403.

\section{How to cite this article:}

Sagar Shashikant Patil, S. S. Balpande, Mohammad Sajid and Pandao, M. R. 2020. Green Synthesis and Characterization of Nano Phosphorus Fertilizer for Wheat. Int.J.Curr.Microbiol.App.Sci. 9(12): 19-23. doi: https://doi.org/10.20546/ijcmas.2020.912.004 